\title{
The Development of a Biofeedback Training System for Cognitive Rehabilitation in Cerebral Palsy
}

\author{
Yu-Luen Chen Chia-Ling Chen** Walter H. Chang* \\ May-Kuen Wong** Fuk-Tan Tung** Te-Son Kuo \\ Department of Electrical Engineering, National Taiwan University \\ Department of Biomedical Engineering, Chung Yuan Christian University* \\ Department of Rehabilitation, Chang Gung Memorial Hospital**
}

\begin{abstract}
A new biofeedback computer-aided biofeedback training system for cognitive rehabilitation in cerebral palsy was developed. It was very difficult for cerebral palsied children to handle entirely in the cognitive rehabilitation protocol. So, in this study, biofeedback training including light, sound, color images and other special functions can induce cerebral palsied children interested in the rehabilitation protocol. Specially, cognitive training is very much important to cerebral palsy which may make the decision of their futures. The purposes of this study are: (1) to develop a computer-based and low cost biofeedback (visual, audio) training system for cognitive rehabilitation. (2) to improve the reaction time and cognitive abilities of cerebral palsied children. At first, the system was tested by twenty normal children to make sure the system basic functions and could work normally. Then, it will apply to cerebral palsied children for future clinical investigation.
\end{abstract}

Keywords: cerebral palsy, biofeedback cognitive rehabilitation, reaction time

\section{Introduction}

Computer-aided and biofeedback training systems have been widely used in rehabilitation for the passed years[1-5].

Human learning begins from new born. Consequently, the earlier the cognitive training, the more plasticity in learning. Although every children have the rights to develop appropriately, but there is limitation in preschool education for children with disabilities (e.g. strict criteria in cases assortment, insufficient case number). Hence, most children with disabilities have no chance to be educated. At present, there is no cognitive educational system for cerebral palsy in most countries, specially, in Taiwan. So, it is desired to develop a cognitive training with Chinese system, and it is very important and emergent to integrate the early cognitive training in rehabilitation. Therefore, in this study, will develop the biofeedback (visual and audio) rehabilitation system for children and the other children with disabilities, specially hand dysfunction. Using this system, the children with disabilities cannot lose their opportunity to learn.

$(0-7803-4262-3 / 97 / \$ 10.00$ (C) 1997 IEEE)

\section{Materials and Methods}

Subjects

In the pilot study, twelve subjects (all boys, age 7-12 years) were selected to make sure the system function could work normally.

\section{Instrumentation}

The new developed system was made of a host computer to control all functions, including output biofeedback signals (sound, light and color images) and each type of training mode to cerebral palsy. The system configuration is shown in figure.1. The main training modes of the system as following and is shown in figure.2:

(a) Hand function control mode

(b) Cognitive training mode

1. Numeral test

2. Fruit images test

3. Cartoon animal images test

4. Actual animal images test

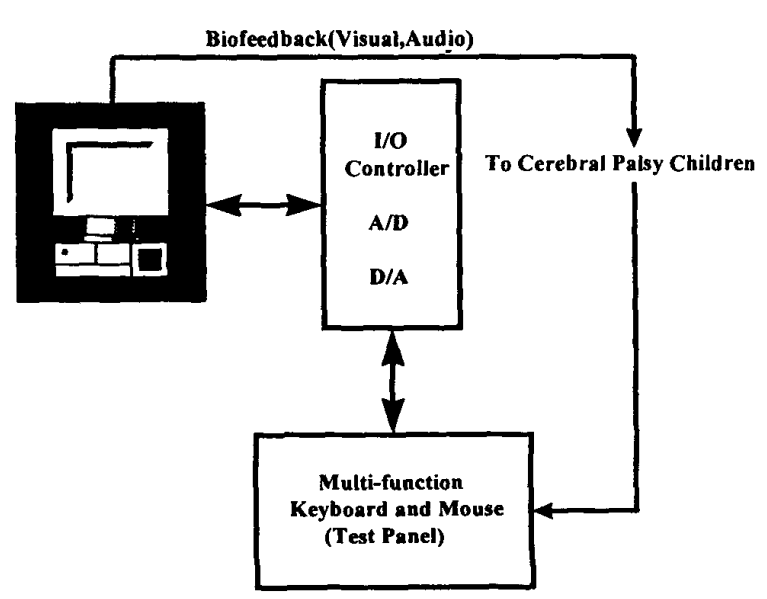

Figure.I The configuration of the cognitive training system 


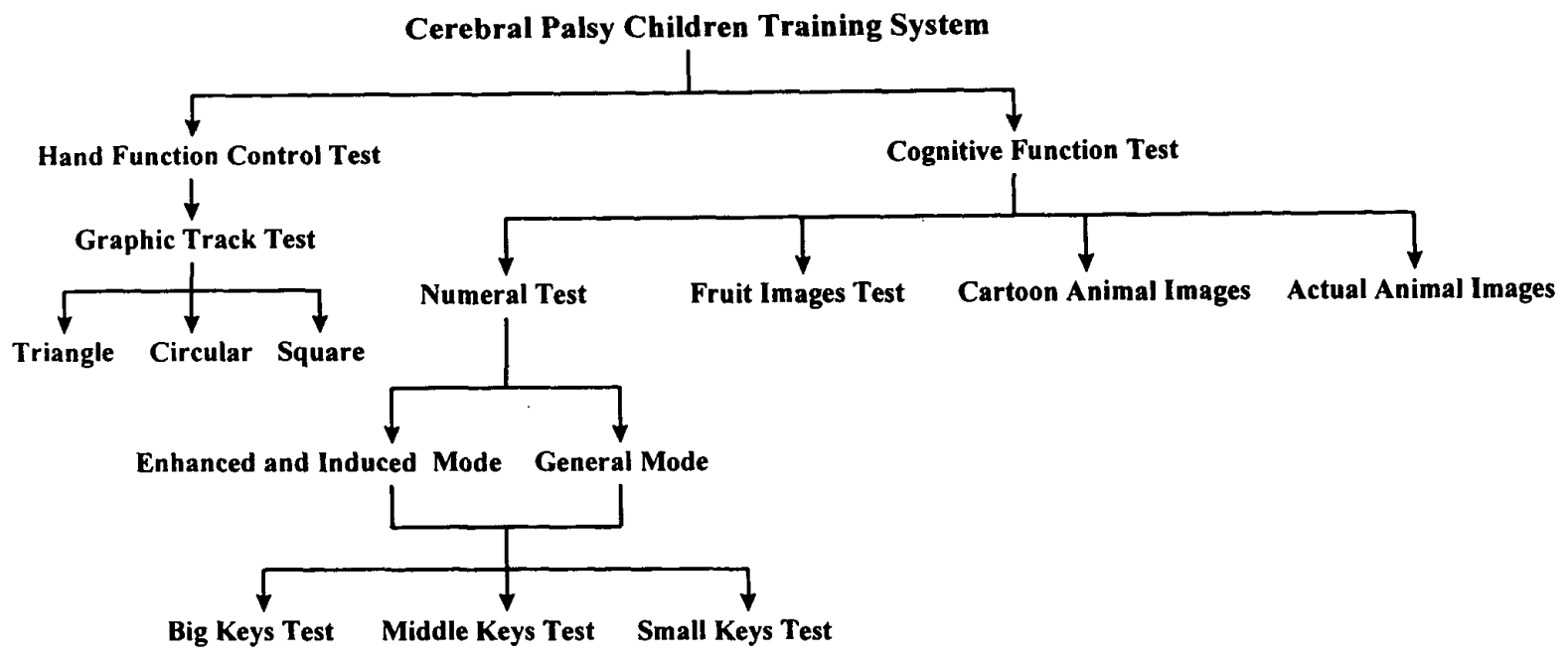

Figure. 2 The functions of the cognitive training system

In the hand function test, triangle, circular and square were included, each shape with a width 1 centimeter (cm). Children can use mouse to control the cursor on the screen, when the cursor maintain in the range of the width of $1 \mathrm{~cm}$ of the shape, then the cartoon music was continued, otherwise, stop the music and a alarm bell were sounded and lighted.

In cognitive training mode, a special keyboard was designed. All keys were made of special switches which contained a light bulb. There were four different tests were included. In each test contained sequencing and random arranging test, and the general mode and enhanced mode were included, respectively. For general test, the screen shown the numbers from 0 to 9 of images step by step. If the key you pressed is the same as shown in the screen then a happy music will sound to you, otherwise, some encourage words displayed and sounded. For enhancement test, the operation step us the same, but when the numbers or images were shown in the screen, the light bulb of relative key will turn on to induce cerebral palsy children to pressed the key. In this test, also can say to learning mode.

\section{Results}

In the pilot study test, the system functions reach to $100 \%$ correction in all tests. It proved the system could work normally. And the clinical assessment will begin to cerebral palsied children.

\section{Conclusion and Discussion}

The new developed cognitive system beside of combination of training and learning, not only cognitive training. For advantage application which could be used to hand functions training of hemiplegic patients. So, the new developed computer-aided cognitive training system through biofeedback has a great potential in rehabilitation.

\section{Acknowledgments}

We thank all staffs of rehabilitation engineering center of Chang Gung Memorial Hospital for clinical support.

\section{Reference}

[1]Caplan B., "Neuropsychology in rehabilitation: its role in evaluation and intervention,". Arch. Phys. Med. Rehabil., vol. 8 pp. 362-366, 1982.

[2]Robertson I.H., Gray J.M., Pentland B., Waite L.J., "Microcomputer-based rehabilitation for unilateral left visual neglect: a randomized controlled trial," Arch. Phys. Med. Rehabil., vol. 9, pp. 663-668, 1990.

[3]Skinner A.D., Trachtman L.H., "Brief or new: use of a computer program (PC Coloring Book) in cognitive rehabilitation," Am. J. Occup. Ther., vol.7, pp. 470-472, 1985.

[4]Levine S.P., Kirsch N.L., Perlman O.Z., Cole T.M., "Engineering therapy: an approach to treatment of a patient with severe cognitive and physical handicaps," Arch. Phys. Med. Rehabil. vol.1 1, pp. 737-739, 1984.

[5]Kirkby K.C., Lambert T.J., "Computer aids to treatment in psychiatry," Australian and New Zealand Journal of Psychiatry, vol. 1, pp. 142-145, 1996. 